\title{
Candida glabrata chorioamnionitis and fungaemia complicating pregnancy following intrauterine insemination
}

Sofie SF Yung1 * FHKCOG, FHKAM (Obstetrics and Gynaecology), Maggie MC Cheng², FHKCOG, FHKAM (Obstetrics and Gynaecology), Paulin WS Ma², FHKCOG, FHKAM (Obstetrics and Gynaecology), PC Ho ${ }^{1}$, MD, FHKAM (Obstetrics and Gynaecology)

${ }^{1}$ Department of Obstetrics and Gynaecology, The University of Hong Kong, Pokfulam, Hong Kong

${ }^{2}$ Department of Obstetrics and Gynaecology, Queen Mary Hospital, Pokfulam, Hong Kong

Hong Kong Med J 2018;24:632-3

*Corresponding author: ssfyung@hku.hk

DOI: 10.12809/hkmj164800

\section{Introduction}

Intrauterine insemination (IUI) is a fertility treatment that involves injection of a processed semen sample into the uterus via a transvaginal catheter after the cervix has been swabbed clean. Sterile instruments are used to reduce the risk of infection.

Historically, Candida glabrata has been considered a relatively non-pathogenic saprophyte of the normal flora of healthy individuals, rarely causing serious infections in humans. Contrary to the more common vaginal coloniser Candida albicans, $C$ glabrata is unable to penetrate intact fetal membranes in vitro. ${ }^{1}$ This explains the rarity of $C$ glabrata chorioamnionitis although it is a common vaginal coloniser found in up to $8 \%$ of pregnant women. ${ }^{1}$

The present study describes a rare case of $C$ glabrata chorioamnionitis and fungaemia in a pregnancy following IUI.

\section{Case presentation}

A 32-year-old healthy primigravida with a trichorionic-triamniotic triplet pregnancy conceived by IUI presented in December 2011 to Queen Mary Hospital, Hong Kong at 16 weeks' gestation with leaking of liquor. Physical examination and ultrasonography confirmed rupture of membranes of one of the triplets. The patient was initially treated with oral erythromycin but 2 days later she developed a fever of $39^{\circ} \mathrm{C}$. Empirical intravenous broadspectrum antibiotics were given for presumptive bacterial chorioamnionitis. In view of the poor prognosis at such an early gestation and the risk of maternal sepsis, medical abortion with misoprostol was considered appropriate. Complete abortion was achieved and the patient's fever subsided the next day.

Two days after abortion, blood culture and vaginal swab test results revealed the presence of $C$ glabrata. Intravenous anidulafungin $200 \mathrm{mg}$ stat, then $100 \mathrm{mg}$ every 24 hours was prescribed. The patient remained well and was discharged from the hospital after 2 weeks of antifungal treatment. Results from a second blood culture taken 3 days after abortion were negative.

In view of the rarity of fungaemia in immunocompetent hosts, testing for human immunodeficiency virus antibodies, lymphocyte subset and dihydrorhodamine reduction test was performed. All results were normal. Histological examination of the three placentae showed polymorph infiltration and presence of fungal spores, confirming acute fungal chorioamnionitis.

\section{Discussion}

To the best of our knowledge, this is the first report of $C$ glabrata chorioamnionitis and fungaemia in a pregnancy following IUI. A search of the literature revealed 18 articles reporting 22 cases of $C$ glabrata chorioamnionitis (online supplementary Appendix $1)$. Fifteen of the reported cases were associated with in-vitro fertilisation (IVF) and four with a foreign body (intrauterine contraceptive device or cervical cerclage). The growing number of reports may be partly due to improved diagnostic methods, but the increasing use of assisted reproductive technologies also appears to be an important factor. Contamination of the embryo by infected semen or the mother's cervicovaginal Candida during transvaginal oocyte harvest, or direct inoculation of $C$ glabrata into the uterus during embryo transfer are possible aetiological mechanisms. ${ }^{2}$ The only two reported cases of the rare Candida lusitaniae chorioamnionitis in the literature were also associated with IVF (online supplementary Appendix 2), further supporting the hypothesis.

Most of the reported cases were associated with preterm labour or preterm premature rupture of membranes (PPROM); therefore, it is also possible that intrauterine $C$ glabrata infection was a result of ascending infection after PPROM and preterm labour, since assisted reproductive technologies are associated with multiple pregnancy, preterm labour, and PPROM. However, PPROM and preterm labour occur in about $6 \%$ of births and there have been only 
23 reported cases (including the current report) of $C$ glabrata chorioamnionitis. Sixteen of the 23 reported cases were associated with IVF and IUI. We conclude that $C$ glabrata chorioamnionitis is more likely due to inoculation of the fungus into the uterus during IVF and IUI procedures, rather than a result of preterm labour and PPROM. The present case supports this hypothesis.

Although maternal outcome was good in the published cases, this fungal chorioamnionitis was often associated with poor fetal outcome. Only $30 \%$ (7 of 23 cases, including the present case) of the published affected pregnancies had any neonatal survival as they usually presented with PPROM or preterm labour in the second or early third trimester.

There are no guidelines for screening for vaginitis before assisted reproductive procedures. If asymptomatic $C$ glabrata or other fungal colonisation is identified, there is no consensus on whether treatment should be given. One case of a successful IVF pregnancy following eradication of $C$ glabrata by topical boric acid treatment had been preceded by an IVF pregnancy that ended in $C$ glabrata sepsis and fetal loss. ${ }^{3}$ The authors suggested routine vaginal culture for yeast and treatment of positive culture results prior to embryo transfer, and commented that such an inexpensive test adds little to the overall costs and procedures associated with IVF. $^{3}$ On the contrary, some authors suggest that a comprehensive screening programme may not be justified given the rarity of invasive disease despite widespread use of IVF and the relatively high rates of $C$ glabrata carriage among pregnant women. ${ }^{2}$ The true incidence of $C$ glabrata chorioamnionitis is unknown because it is likely to be underdiagnosed due to the difficulty in laboratory diagnosis. ${ }^{2}$ Treatment cost-effectiveness cannot be estimated, but the number needed to treat to prevent one $C$ glabrata-related adverse outcome is likely to be large in view of the relatively high carrier rate and the rarity of $C$ glabrata chorioamnionitis. Further research is needed to evaluate the cost-effectiveness of such a screen-and-treat programme before it can be recommended. In addition, there have been reports of emergence of antifungal resistance. If all asymptomatic carriers are treated prior to embryo transfer, emergence of drug resistance will become a concern. Similarly, whether it is worth screening men for any asymptomatic carriage is uncertain.

\section{Conclusion}

The possibility of $C$ glabrata chorioamnionitis should be considered in pregnant women who develop chorioamnionitis after conceiving with assisted reproductive techniques. The reporting of this potentially devastating condition should be encouraged so as to improve our knowledge about its incidence, risk factors, pathogenesis, and management. Further research is needed to determine the incidence of fungal infectionrelated adverse outcomes and the cost-effectiveness of a screen-and-treat approach prior to assisted reproduction.

\section{Author contributions}

All authors contributed to the concept of the paper. SSF Yung, MMC Cheng, and PWS Ma contributed to the acquisition, analysis, and interpretation of data. SSF Yung drafted the article.

\section{Declaration}

All authors have disclosed no conflicts of interest. All authors had full access to the data, contributed to the study, approved the final version for publication, and take responsibility for its accuracy and integrity. This paper was presented as a poster in the 25th Asian \& Oceanic Congress of Obstetrics and Gynaecology (AOCOG), Hong Kong, on 15-18 June 2017.

\section{References}

1. Ganer Herman H, Mevorach Zussman N, Krajden Haratz K, Bar J, Sagiv R. Candida glabrata chorioamnionitis following in vitro fertilization: review of the literature. Gynecol Obstet Invest 2015;80:145-7.

2. Jackel D, Lai K. Candida glabrata sepsis associated with chorioamnionitis in an in vitro fertilization pregnancy: case report and review. Clin Infect Dis 2013;56:555-8.

3. Asemota OA, Nyirjesy P, Fox R, Sobel JD. Candida glabrata complicating in vitro pregnancy: successful management of subsequent pregnancy. Fertil Steril 2011;95:803.e1-2. 